\title{
Pneumatic Injection Therapy-Induced Transcutaneous Penetration of Hypertonic Glucose Solution: Macro- and Microscopic Analyses of Human and Rat Tissues
}

\author{
Hyun-Jo Kim ${ }^{1}$ \\ Seung-Ho Han² \\ A Young Park ${ }^{3}$ \\ Heesu Kim ${ }^{4}$ \\ Gi Woong Hong ${ }^{5}$ \\ Ee Seok Lim ${ }^{6}$ \\ Sung Bin $\mathrm{Cho}^{4,7}$
}

\author{
${ }^{1}$ CNP Skin Clinic, Cheonan, Korea \\ ${ }^{2}$ Department of Anatomy, Chung-Ang University \\ College of Medicine, Seoul, Korea \\ ${ }^{3}$ Department of Dermatology, Soonchunhyang \\ University College of Medicine, Cheonan, Korea \\ ${ }^{4}$ Department of Dermatology and Cutaneous \\ Biology Research Center, International St. Mary's \\ Hospital, Catholic Kwandong University College of \\ Medicine, Incheon, Korea \\ ${ }^{5}$ Samskin Plastic Surgery, Seoul, Korea \\ ${ }^{6}$ Thema Skin Clinic, Seoul, Korea \\ ${ }^{7}$ Kangskin Dermatology Clinic, Seoul, Korea
}

Received June 2, 2018

Accepted June 11, 2018

Correspondence
Sung Bin Cho
Department of Dermatology and Cutaneous
Biology Research Center, International St. Mary's
Hospital, Catholic Kwandong University College of
Medicine, 25 Simgok-ro, Seo-gu, Incheon 22711,
Korea
Tel.: +82-32-290-3141
Fax: +82-32-290-3142
E-mail: drsbcholagmail.com
(C) Korean Society for Laser Medicine and Surgery
@ This is an open access article distributed under the
terms of the Creative Commons Attribution Non-
Commercial License (http://creativecommons.org/
licenses/by-nc/4.0) which permits unrestricted non-
commercial use, distribution, and reproduction in any
medium, provided the original work is properly cited.

\author{
Background and Objectives \\ Pneumatic injection therapy (PIT) is a needle-free, penetration- \\ enhanced, transdermal method of delivering drugs into the skin. This \\ study evaluated topographically the PIT-induced transcutaneous \\ infiltration of a $20 \%$ hypertonic glucose solution in fresh human cadavers \\ and outlined the microscopic patterns and reactions to PIT with a $20 \%$ \\ glucose solution in in vivo rat skin.
}

\section{Materials and Methods}

The penetration depths of a $20 \%$ glucose solution by PIT were analyzed macroscopically in 10 hemifaces from fresh cadavers. In addition, the infiltration patterns of PIT with a $20 \%$ glucose solution performed in vivo in rats were analyzed through a histology evaluation.

\section{Results}

Pneumatic injections of a hypertonic glucose solution generated PIT zones in the subcutaneous fat and superficial temporal fascia layers (10 out of 10 hemifaces), superficial layer of the deep temporal fascia (DTF; 8 out of 10 hemifaces), and deep layer of the DTF (7 out of 10 hemifaces). In addition, distinctive PIT zones were generated in rat tissues from the upper papillary deep to the muscle and fascia layers.

\section{Conclusion}

The data suggest that PIT-induced, jet infiltration of a $20 \%$ hypertonic glucose solution contributes to clinical improvement of skin laxity by stimulating the wound repair processes and increasing collagen production throughout multiple layers of the skin.

\section{Key words}

Transcutaneous pneumatic injection; Prolotherapy; Hyperglycemic solution; Cadaver; Rat; Ultrasound 


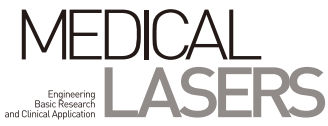

\section{INTRODUCTION}

Prolotherapy refers to perilesional injection therapy of hypertonic glucose solution to induce a hyperglycemic state for treating chronically painful musculoskeletal conditions. ${ }^{1,2}$ The hyperglycemic condition induces wound repair processes marked by infiltration of inflammatory cells; moreover, phenotypical activation of fibroblasts is achieved by mechanical loading of infiltrated solution. ${ }^{3-8}$ Thus, the suggested action mechanisms of prolotherapy include the induction of fibroblast proliferation, collagen deposition, and vascular proliferation, as well as the thickening of ligamentous structures. ${ }^{1,2,9,10}$

Pneumatic injection therapy (PIT) is a needle-free, penetration-enhanced, transdermal method of delivering drugs into targeted areas of the skin. ${ }^{11,12}$ A variety of solutions, including normal saline, $5 \%$ or $20 \%$ glucose solution, botulinum toxins, and soft tissue fillers, can be forcefully, but with minimal invasiveness, injected into the skin and subdermal layers, as well as the superficial musculoaponeurotic system (SMAS) or muscle layers, depending on the pressure settings. ${ }^{11-15}$ By doing so, PIT characteristically generates semi-circular or cylindrical zones of tissue infiltration and leaves pin-point entry points in the epidermis. ${ }^{11,12}$ Our study group previously compared PIT-induced tissue reactions with 5\% isotonic and $20 \%$ hypertonic glucose solutions in in vivo micropig skin and tissue-mimicking phantom. ${ }^{12}$ PIT of $20 \%$ hypertonic glucose immediately generated smaller, homogeneous glucose droplets in the dermis, and elicited more remarkable post-procedural inflammatory cell infiltration, compared to PIT of $5 \%$ isotonic glucose solution. ${ }^{12}$

In this study, we aimed to topographically evaluate PITinduced transcutaneous penetration of $20 \%$ hypertonic glucose solution in human fresh cadavers. The layer-dependent infiltration patterns were analyzed immediately after PIT by dissecting the temple of the cadavers. The order of dissection thereof was as follows: the skin, subcutaneous fat, superficial temporal fascia (STF), and the superficial and deep layers of the deep temporal fascia (DTF). Immediate in vivo human tissue reactions by PIT were also evaluated using two-dimensional ultrasound. Additionally, in the aforementioned in vivo experimental study using micropig skin, we sought to compare the infiltration patterns of different injectable solutions; however, the depths of infiltration over the dermosubcutaneous junction were significantly limited by thick dermal collagen bundles. ${ }^{12}$ Therefore, we also performed an in vivo study in experimental rats to evaluate the patterns of and reactions to infiltration of $20 \%$ hypertonic glucose solution into the subcutaneous fat and fibromuscular layers.

\section{MATERIALS AND METHODS}

\section{Pneumatic injection therapy system and inject- able solution}

A PIT device (Shemax ${ }^{\top M}$; Shenb Co., Ltd., Seoul, Korea) that delivers compressed pneumatic pressure to a disposable nozzle filled with injectable solution was utilized in this study. Depending on the therapeutic purposes and the targeted injection sites, the pneumatic pressure can be adjusted as described in a previous report. ${ }^{12}$ The filling volume of injectable solution is determined by regulating the opening duration of the nozzle. Additionally, 20\% hypertonic glucose solution (Huons, Bundang, Korea) was used as an injectable solution, and was pretreated with $5 \%$ (v/v) methylene blue for macroscopic visualization.

\section{Pneumatic injection therapy on human cadaveric tissue}

To evaluate the penetration depths of the hypertonic glucose solution, 10 hemifaces from five legally donated, fresh cadavers ( 4 males and 1 female; median age: 62 years; age range: 59-65 years) were macroscopically analyzed in this study. Each hemiface was treated with a single pass of PIT on the skin of the temple area. Approximately 30 PIT shots at intervals of $1-2 \mathrm{~cm}$ were delivered to each hemiface. The trigger point injection settings comprised a pneumatic pressure of 4.13 bars and an injection volume of $0.08 \mathrm{ml}$, with a nozzle opening duration of $57 \mathrm{msec} / \mathrm{shot}$. Immediately after the jet infiltration of hypertonic glucose solution, the temple areas of the cadaveric skin were dissected gently in the following order: the skin, subcutaneous fat, STF, and superficial and deep layers of the DTF. PIT zones in each anatomic layer were measured using Image J software Version 1.48; National Institutes of Health, Bethesda, MD, USA), and the relative areas thereof, which were calculated in reference to the area of PIT zones in the subcutaneous fat layer, are presented as means \pm standard deviation.

\section{Ultrasound examination}

Immediate in vivo human tissue reactions at various pressure settings were evaluated using two-dimensional ultrasound study in a 40-year-old male volunteer. After obtaining written informed consent, a single pass of PIT was made along the non-hair-bearing area of the frontal hairline and abdominal skin after cleansing with 70\% ethanol in the supine position without topical or systemic anesthesia. PIT was performed with the pneumatic pres- 
sure settings of $2.05,2.55,3.15,3.63,4.13$, and 4.64 bars; an injection volume of $0.12 \mathrm{ml} / \mathrm{shot}$; and a nozzle opening duration of $61 \mathrm{msec} / \mathrm{shot}$. Each shot was spaced at least $2 \mathrm{~cm}$ from the others to avoid overlapping effects from other experimental conditions. Immediately after PIT, two-dimensional ultrasound (ECUBE15; Alpinion Medical Systems Co., Ltd., Seoul, Koreal was emitted at the entry points and treatment sites to evaluate the characteristic features of the infiltrated skin layers. Two-dimensional ultrasound images were obtained using a linear highfrequency hockey stick transducer (I08-17; Alpinion) at a frequency of 8-17 MHz, measuring maximally $3 \mathrm{~cm}$ deep for the frontal hairline and $5 \mathrm{~cm}$ deep for the abdomen. The heights and widths of PIT zones are presented as means \pm standard deviation. This study was approved by the Institutional Review Board of International St. Mary's Hospital, Catholic Kwandong University College of Medicine.

\section{Pneumatic injection therapy on in vivo rat tissue}

All experimental protocols were approved by the ethics committee of the Soonchunhyang University Institutional Animal Care and Use Committee. Eight male, SpragueDawley rats were purchased (Orient Bio Corp., Seongnam, Koreal at the age of 6 weeks, and the in vivo experiments were performed at the age of 14 weeks at weights of $450-500 \mathrm{~g}$. After cleansing with a mild soap and 70\% alcohol, each rat was treated with a single pass of PIT along the back skin, with a total shot count of 30 shots at intervals of 1-2 cm, under ether anesthesia. The trigger point injection settings comprised a pneumatic pressure of 3.63 bars, an injection volume of $0.06 \mathrm{ml}$ of $20 \%$ hypertonic glucose solution, and a nozzle opening duration of $55 \mathrm{msec} / \mathrm{shot}$.

\section{Histologic evaluation}

The experimental rats were sacrificed for sampling the treated tissue in a humane manner according to standard protocols. Immediately and 1, 2, and 3 weeks after treatment, full thickness tissue specimens, including the epidermis, dermis, subcutaneous fat, muscle, and fascia, were obtained for microscopic evaluation. Each sample was fixed in 10\% buffered formalin and embedded in paraffin. Then, serial tissue sections of 4- $\mu$ m thickness for each treatment setting were prepared and stained with hematoxylin and eosin and Masson's trichrome.

\section{RESULTS}

\section{Pneumatic injection therapy on human cadaveric tissue}

Immediately after PIT, bluish, uniform, pin-point entry points were found in the epidermis in all cadaveric samples (Fig. 1A). Approximately 1-cm, skin-colored papules were generated around the entry points. After dissecting the epidermis, dermis, and subcutaneous fat, round to
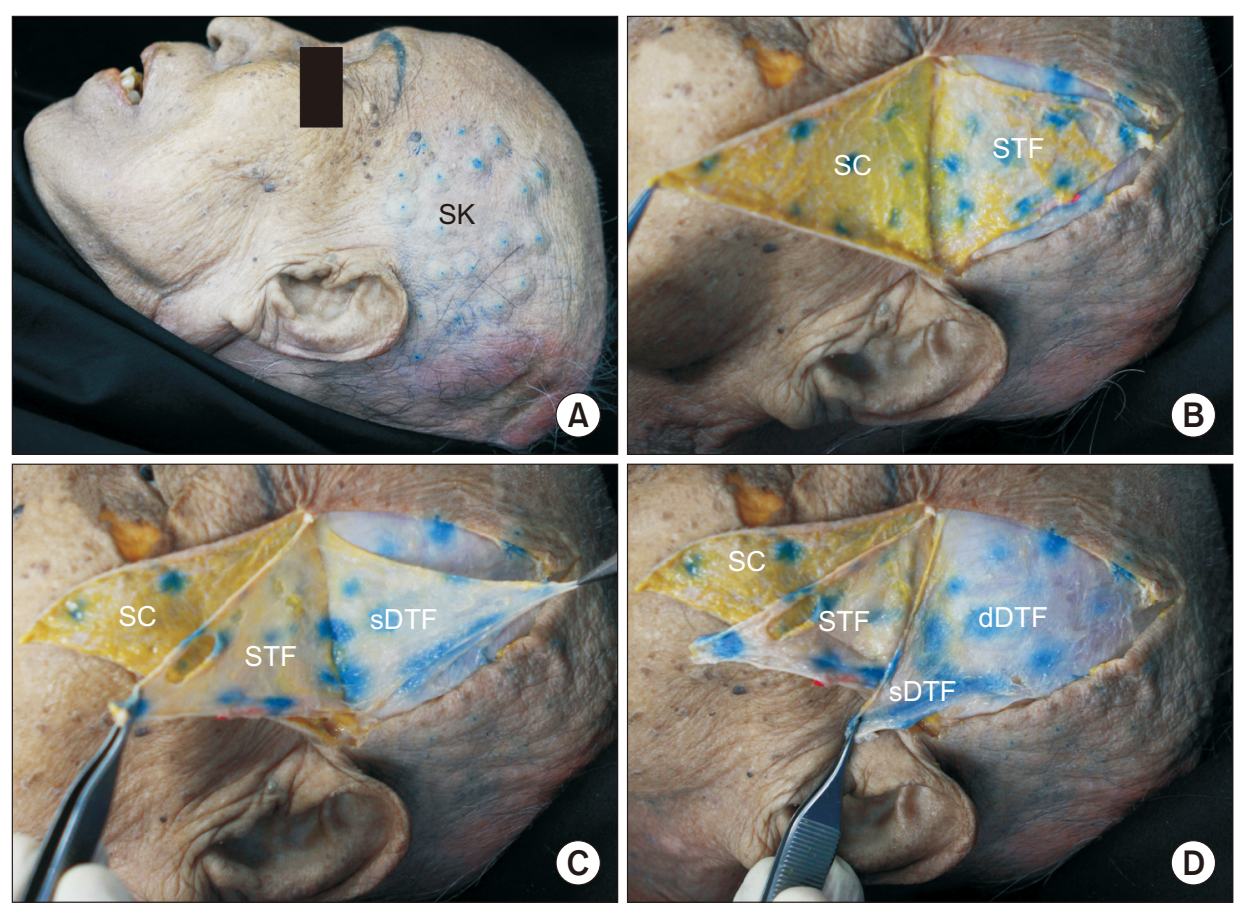

Fig. 1. Pneumatic injection therapy (PIT) on human cadaveric tissue. (A) PIT-induced bluish pin-point entry points on the epidermis. The PIT zones of $20 \%$ hypertonic glucose solution (B) in the subcutaneous fat (SC) layer and superficial temporal fascia (STF), (C) the superficial layer of the deep temporal fascia (sDTF), and (D) the deep layer of the deep temporal fascia (dDTF). SK, skin. 
oval zones of bluish 20\% hypertonic glucose solution were found in the subcutaneous fat layer in all hemifaces (Fig. 1B). Moreover, the bluish PIT zones were also found in the STF in all hemifaces, and the relative mean area thereof was remarkably larger $(2 \pm 0.5)$ than the infiltrated zones in the subcutaneous fat layer ( $1 \pm 0.7$ ) (Fig. 2).

After removing the STF, the DTF was identified and further divided into the superficial and deep layers. In the superficial layer of the DTF, bluish PIT zones were found in eight (80\%) of 10 hemifaces, with a relative mean area of $3.3 \pm 1.2$ (Fig. 1C). Moreover, the zones of glucose infiltration were found in seven (70\%) of 10 hemifaces in the deep layer of the DTF, with a relative mean area of $2.2 \pm 0.8$ (Fig. 1D). Among the seven hemifaces wherein glucose infiltrated the deep layer of the DTF, the greatest number

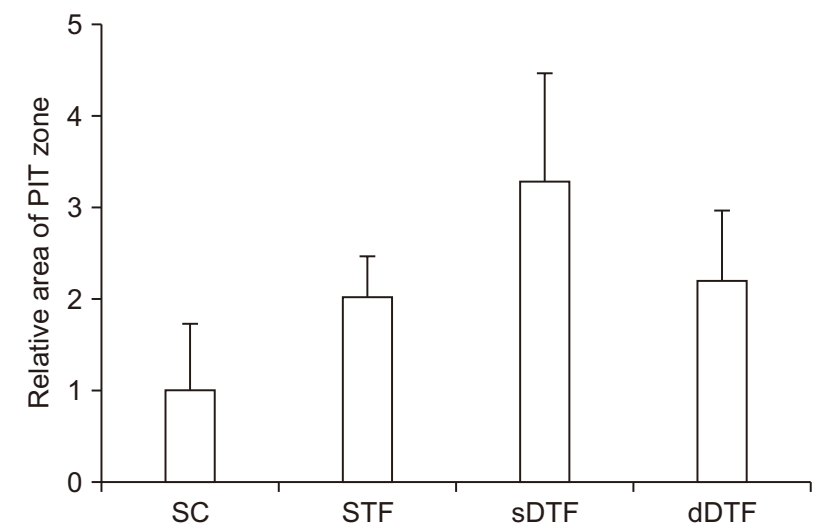

Fig. 2. Relative areas of PIT zones in human cadaveric tissue. The values are presented as means \pm standard deviation. The relative area of PIT zones in each of the STF, sDTF, and dDTF was calculated in reference to the PIT area in the subcutaneous fat layer. of infiltrated zones was found in the subcutaneous fat layer, disregarding the epidermis where the solution entered the skin, and the fewest was observed in the deep layer of the DTF. Meanwhile, the largest zones of glucose infiltration were generated in the superficial layer of the DTF, and the smallest zones were found in the epidermis.

\section{Immediate tissue reactions on two-dimensional ultrasound}

Immediately after applying PIT with 20\% hypertonic glucose solution on in vivo human tissue, the pressureand site-dependent features of tissue infiltration were evaluated by two-dimensional ultrasound examination. To do so, PIT was delivered on the non-hair-bearing area of the frontal hairline and abdominal skin to obtain ultrasound images free from interference by hair shafts. The PIT settings of 2.05 bars and $0.12 \mathrm{ml} /$ shot elicited well-demarcated, heterogeneous infiltration of glucose solution throughout the dermis and in the dermosubcutaneous fat junction (mean height, $3.5 \pm 0.5 \mathrm{~mm}$; mean width, 9.2 $\pm 0.4 \mathrm{~mm}$ ), with remarkable postacoustic shadowing (Fig. 3A). The settings of 2.55 bars and $0.12 \mathrm{ml} /$ shot also generated well-demarcated, heterogeneous, oval PIT zones throughout the dermis and subcutaneous fat layer (mean height, $5.1 \pm 0.4 \mathrm{~mm}$; mean width, $14 \pm 1.9 \mathrm{~mm}$ ) (Fig. 3B). Moreover, the central and superficial parts of PIT zones, which surrounded the entry points, showed hyperechogeneous infiltration, compared to the peripheral and deeper parts of PIT zones, in the settings of both 2.05 and 2.55 bars.

At the pressure settings of $3.15,3.63$, and 4.13 bars and an injection volume of $0.12 \mathrm{ml} / \mathrm{shot}$, well-demarcated,
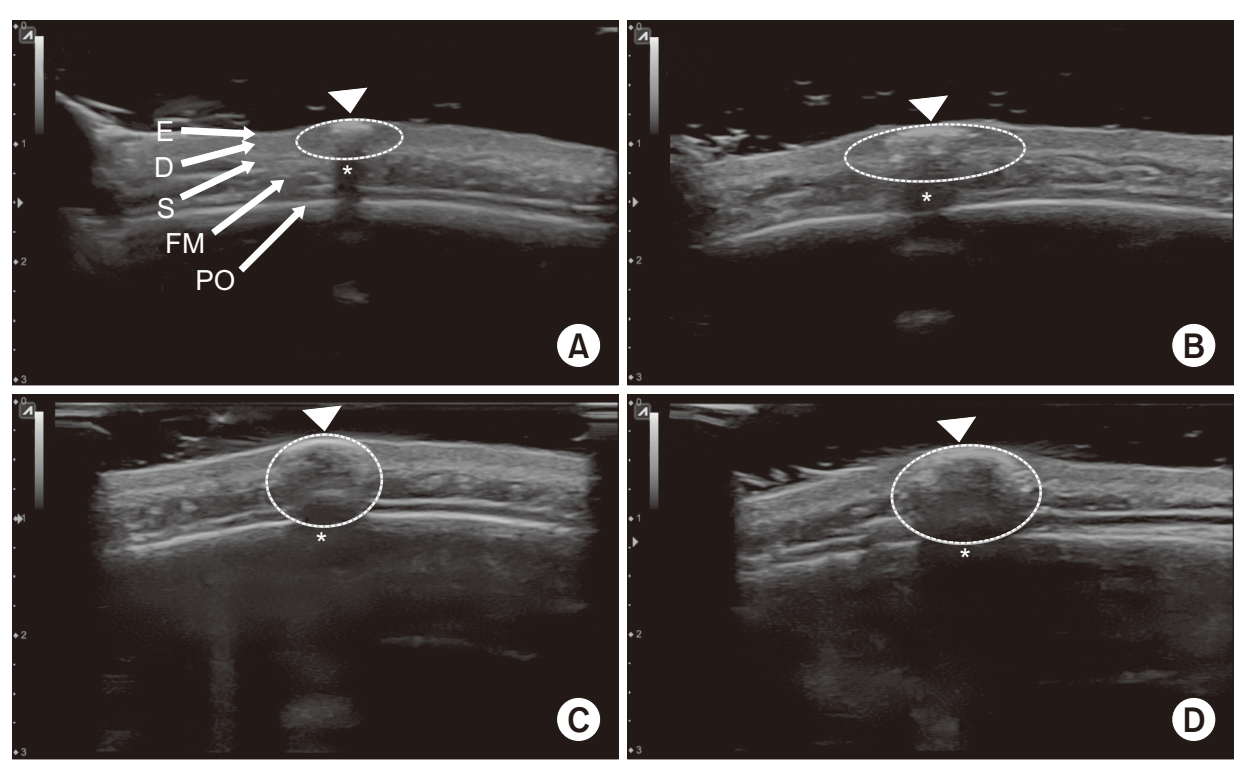

Fig. 3. Ultrasound examination. Two-dimensional ultrasound images exhibiting PIT zones (white circles) immediately after PIT on the nonhair-bearing area of the frontal hairline using $20 \%$ hypertonic glucose solution in in vivo human. The PIT settings included an injection volume of $0.12 \mathrm{ml} / \mathrm{shot}$ and a pressure of (A) 2.05 bars, (B) 2.55 bars, (C) 3.15 bars, and (D) 3.63 bars. A linear high-frequency hockey stick transducer at a frequency of 8-17 $\mathrm{MHz}$. Asterisks indicate postacoustic shadowing. E, epidermis; D, dermis; S, subcutaneous fat layer; FM, fascia and muscle; PO, periosteum. 
heterogeneous, round to oval PIT zones were observed at the levels of the dermis, subcutaneous fat, facial muscle and fascia, and periosteum (Fig. 3C, D, and 4A). Meanwhile, however, the PIT zones became wider and deeper with higher pneumatic pressures $(3.15$ bars, mean height of $7.6 \pm 0.2 \mathrm{~mm}$ and mean width of $9.6 \pm 0.2 \mathrm{~mm} ; 3.63$ bars, mean height of $8.6 \pm 0.2 \mathrm{~mm}$ and mean width of 12.4 $\pm 0.6 \mathrm{~mm} ; 4.13$ bars, mean height of $8.3 \pm 0.2 \mathrm{~mm}$ and mean width of $11.5 \pm 0.5 \mathrm{~mm}$ ). Additionally, PIT on abdominal skin at the pressure settings of $3.63,4.13$, and 4.64 bars and an injection volume of $0.12 \mathrm{ml} / \mathrm{shot}$ exhibited narrower, but deeper infiltration patterns of $20 \%$ glucose solution, compared to PIT on the frontal hairline (Fig. 4BD). The PIT zones showed a mean height of $17.5 \pm 0.3 \mathrm{~mm}$ and a mean width of $14.5 \pm 0.6 \mathrm{~mm}$ at the pressure of 3.63 bars, a mean height of $16.8 \pm 0.2 \mathrm{~mm}$ and a mean width of $15.8 \pm 0.5 \mathrm{~mm}$ at 4.13 bars, and a mean height of $15.1 \pm 0.6$ $\mathrm{mm}$ and a mean width of $18.1 \pm 0.1 \mathrm{~mm}$ at 4.63 bars.

\section{Pneumatic injection therapy on in vivo rat tissue}

Immediately after PIT with 20\% hypertonic glucose solution at the injection volume of $0.06 \mathrm{ml} / \mathrm{shot}$ and a pneumatic pressure of 3.63 bars, distinctive PIT zones were generated in rat tissues from the upper papillary deep to the muscle and fascia layers (Fig. 5A-C). Each PIT zone was composed of numerous round to oval droplets that surrounded entry points and of sigmoid-shaped central droplets. The surrounding glucose droplets in the mid and lower dermis and subcutaneous fat tissue were larger in size, compared to those in the upper dermis. Furthermore, the glucose droplets infiltrated extensively into the superficial fascia layer, and several droplets were found in the muscular layer (Fig. 5D-F). In the deep fascia layer, the glucose solution infiltrated by penetrating through the fibrous septum of skeletal muscles.

At one week post-PIT, glucose droplets were not observed in any layers of the rat skin. Collagen fibers in the dermis, the fibrous septum of the subcutaneous fat and skeletal muscle, and the superficial and deep fascia layers were fragmented and irregularly arranged, but were thicker than in controls (Fig. 6A, D). At two weeks postPIT, we noted marked increases in thickened collagen fibers in all layers of the rat specimens (Fig. 6B, E). At three weeks post-PIT, the amounts of the thicker collagen bundles had remarkably increased in the dermis, subcutaneous fat, and superficial fascia layers, compared to control, 1 week post-PIT, and 2 weeks post-PIT specimens (Fig. 6C, F).

\section{DISCUSSION}

In this study, we aimed to topographically evaluate the patterns of PIT-induced, layer-dependent, glucose infiltration immediately after delivering PIT on the temporal scalp. To do so, we obtained horizontal macroscopic views of the layers of fresh cadaveric tissues by gently dissecting each layer of the skin and subcutaneous fat tissue, STF, and superficial and deep layers of the DTF.

In previous cadaveric study, injectable solutions were shown to generate semi-circular PIT zones throughout the epidermis, dermis, subcutaneous fat, and SMAS layers at the pressure setting of 6 bars. ${ }^{11}$ The size of PIT zones was deemed to be greatest in the SMAS, followed by the subcutaneous fat tissue and upper part of the der-
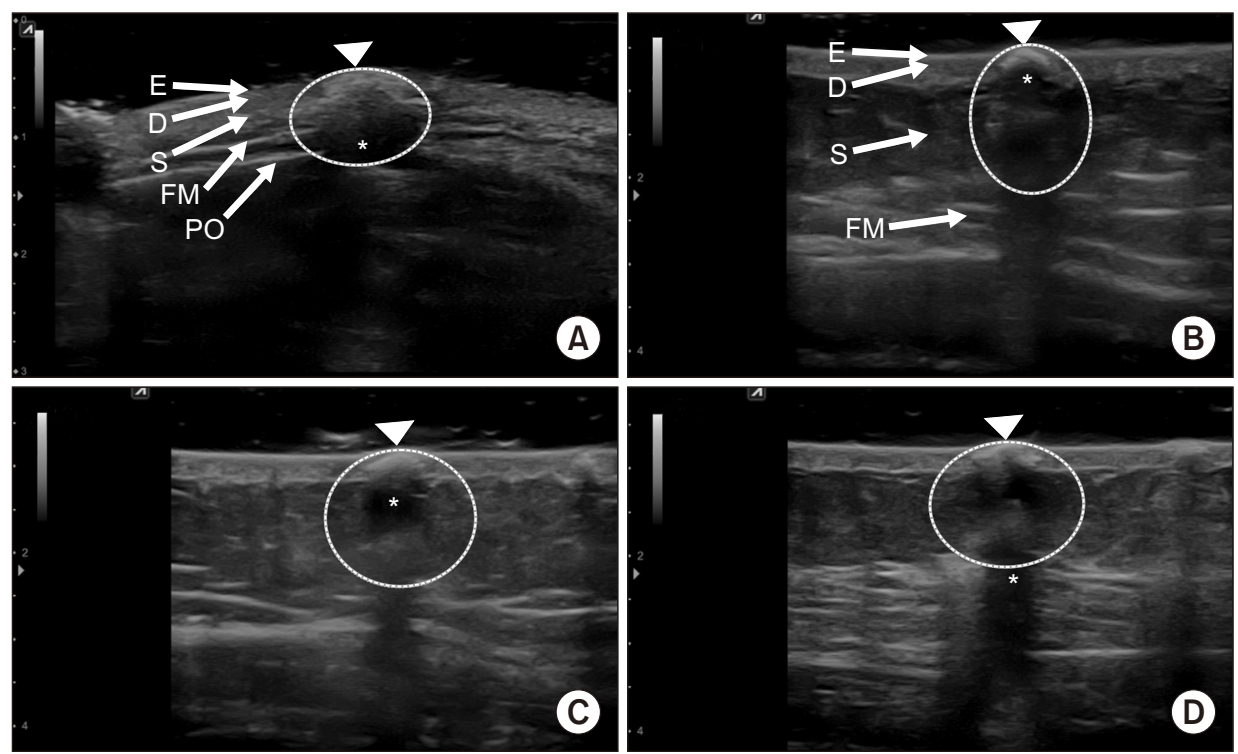

Fig. 4. Ultrasound examination. Two-dimensional ultrasound images demonstrating PIT zones (white circles) immediately after PIT on the non-hair-bearing area of the (A) frontal hairline and (B-D) abdomen using 20\% hypertonic glucose solution in in vivo human. The PIT settings included an injection volume of $0.12 \mathrm{ml} / \mathrm{shot}$ and a pressure of (A) 4.13 bars, (B) 3.63 bars, (C) 4.13 bars, and (D) 4.64 bars. A linear high-frequency hockey stick transducer at a frequency of 8-17 MHz. Asterisks indicate post-acoustic shadowing. E, epidermis; D, dermis; $\mathrm{S}$, subcutaneous fat layer; FM, fascia and muscle; PO, periosteum. 

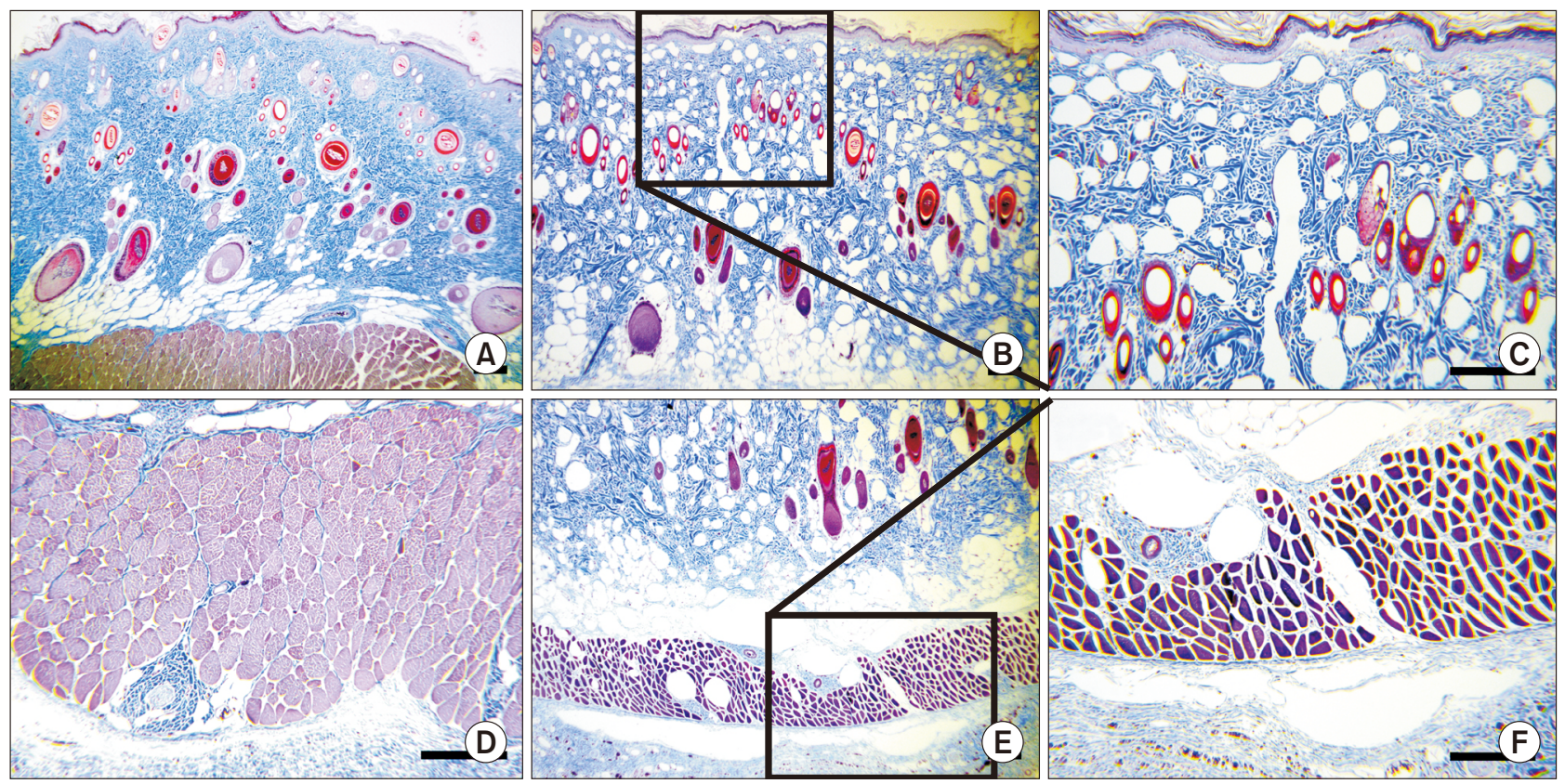

Fig. 5. Immediate PIT-induced tissue reactions in in vivo rat tissue. (A, D) Untreated rat tissue. (B, C, E, F) Immediate PIT-induced tissue reactions in in vivo rat tissue after the jet-infiltration of $20 \%$ hypertonic glucose solution. (B, E) Inlets show magnified areas of (C) the epidermis and dermis and (F) the fibromuscular layers. Masson's trichrome stain, original magnification (A, B, E) $\times 40$ and (C, D, F) $\times 100$, scale bar $=200 \mu \mathrm{m}$.
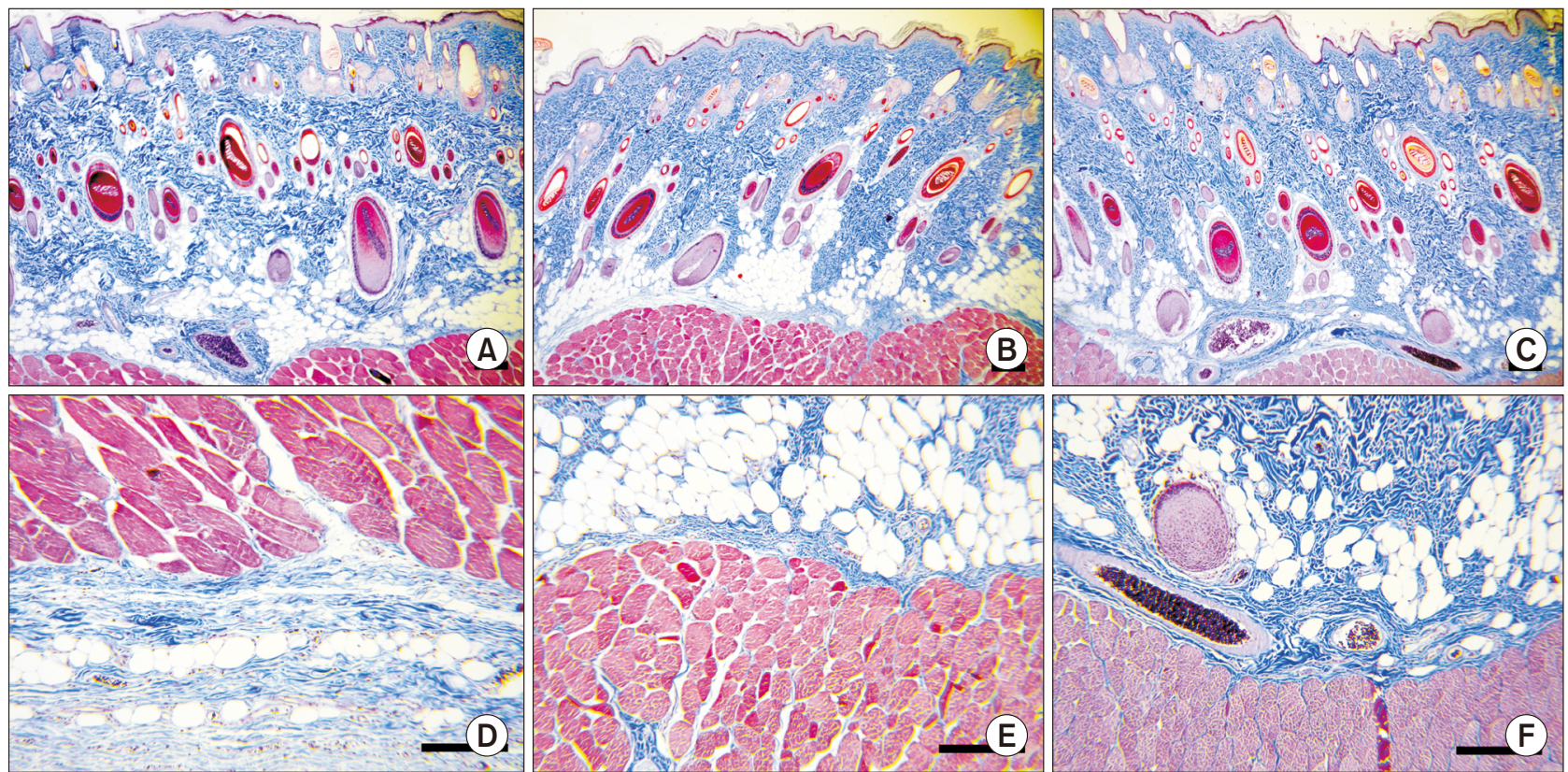

Fig. 6. Delayed PIT-induced tissue reactions in in vivo rat tissue. Post-PIT tissue reactions in the epidermis, dermis, subcutaneous fat, and fibromuscular layers at (A, D) one week, (B, E) two weeks, and (C, F) three weeks after treatment. Masson's trichrome stain, original magnification $(\mathrm{A}-\mathrm{C}) \times 40$ and $(\mathrm{D}-\mathrm{F}) \times 100$, scale bar $=200 \mu \mathrm{m}$.

mis. ${ }^{11}$ Meanwhile, PIT at an 8.5-bar pressure setting histologically exhibited cylindrical infiltration zones throughout the epidermis, dermis, subcutaneous fat, SMAS, and masseter muscle layers. Thereby, the size of PIT zones were deemed uniform throughout the infiltrated layers. ${ }^{11}$

In our cadaveric study, distinctive PIT zones were mac- 
roscopically found in the epidermis, dermis, subcutaneous fat, STF, and DTF layers at the pressure setting of 4.13 bars. The layered dissection of post-PIT cadaveric tissues revealed the generation of greater PIT zones in the STF and DTF in most of the cases, compared to the subcutaneous fat layer. We suggest that the relatively lower pressure setting could effectively reach target layers of the STF and DTF during face lifting; however, the degrees and patterns of infiltration differed in individual cadaveric tissues.

In this study, two-dimensional ultrasound examination exhibited well-demarcated PIT zones of tissue infiltration immediately after PIT using 20\% glucose solution in in vivo human tissue. At lower-pressure settings, such as 2.05 and 2.55 bars, in the frontal hairline, PIT zones were observed throughout the epidermis and dermis with central hyperechogenicity, and the largest area of tissue infiltration was found in the mid portions of the dermis. Meanwhile, at higher pressure settings, such as 3.15, 3.63 , and 4.13 bars, in the frontal hairline, PIT zones were generated throughout the skin, subcutaneous fat, muscle and fascia, and periosteum layers with peripheral hyperechogenicity. Moreover, the largest relative area of tissue infiltration was found deeper in the subcutaneous fat or muscle and fascia layers at higher pressure settings.

Our previous in vivo micropig study compared the infiltration patterns of $5 \%$ isotonic and $20 \%$ hypertonic glucose solutions injected with PIT. ${ }^{12}$ Therein, PIT zones generated by $20 \%$ glucose solution were composed of small, homogeneous glucose droplets in the dermis, whereas $5 \%$ glucose solution generated PIT zones of heterogeneous glucose droplets of varying sizes in the dermis. ${ }^{12}$ Unfortunately, the thick collagen bundles in the dermis of the micropig skin limited the depth of penetration, and as such, the PIT-induced infiltration patterns in the subcutaneous fat, muscle, and fascia layers could not be evaluated. Therefore, in this study, we performed PIT on rat skin, and full-thickness tissue specimens, which contained skin, subcutaneous fat, superficial fascia, skeletal muscle, and deep fascia layers, were evaluated.

The thickness of the temple area, which consists of six or seven layers, decreases with age. ${ }^{16-18}$ The age-related volume loss of the superficial or deep part of fat tissues or temporalis muscle has been suggested to contribute thereto. ${ }^{17-19}$ Because decreased volumes in the topographic components in the temple area are associated with loosening among the skin layers, the laxity of the face can eventually be aggravated. ${ }^{17-19}$ By inducing the wound repair process and tightening among the topographic layers of the temple area, prolotherapy is theoretically effective for the restoration of facial skin laxity. Our microscopic in vivo experimental rat study revealed that PIT-induced jet-infiltration of $20 \%$ glucose solution reached the layers of skeletal muscle and deep fascia. Furthermore, PIT-induced tissue injury and accompanying wound repair resulted in marked increases in collagen production throughout the infiltrated zones over the duration of 3 weeks post-PIT. Accordingly, in light of these results, we propose that PIT-induced wound repair and increased collagen fibers throughout the multilayers of the temporal area elicited by jet-infiltration of $20 \%$ hypertonic glucose solution can effectively contribute to clinical improvement in face lifting.

In conclusion, our topographical study using human fresh cadavers demonstrated that PIT with 20\% glucose solution penetrates to the deep part of the DTF. Moreover, our in vivo human study demonstrated that the injectable solution could be more effectively delivered peripherally and deeply into the target tissue at higher pressure settings. Finally, in vivo experimental rat study revealed that the jet-infiltrated glucose solution penetrates to the deeper fascia layers and stimulates wound repair processes and collagen production. Our findings suggest that PIT jet-infiltration of $20 \%$ hypertonic glucose solution can contribute to clinical improvements in skin laxity by stimulating wound repair processes and increasing collagen production throughout multiple layers of targeted skin tissue.

\section{ACKNOWLEDGEMENTS}

We would like to thank Bora Kim (Shenb Co., Ltd., Seoul, Koreal and Min Choi (Shenb Co.) for their assistance with technical support. We would also like to thank Anthony Thomas Milliken, ELS, at Editing Synthase (https://editingsynthase.com) for his help with the editing of this manuscript.

\section{DISCLOSURE STATEMENT}

No potential conflict of interest was reported by the authors.

\section{REFERENCES}

1. Sit RW, Chung VCh, Reeves KD, Rabago D, Chan KK, Chan DC, et al. Hypertonic dextrose injections (prolotherapy) in the treatment of symptomatic knee osteoarthritis: a systematic review and meta-analysis. Sci Rep 2016;6:25247.

2. Rabago D, Best TM, Beamsley M, Patterson J. A systematic 
review of prolotherapy for chronic musculoskeletal pain. Clin J Sport Med 2005;15:376-80.

3. Sugiura T, Yamauchi A, Kitamura H, Matusoka Y, Horio M, Imai $E$, et al. Effects of hypertonic stress on transforming growth factor-beta activity in normal rat kidney cells. Kidney Int 1998;53:1654-60.

4. Han DC, Isono M, Hoffman BB, Ziyadeh FN. High glucose stimulates proliferation and collagen type I synthesis in renal cortical fibroblasts: mediation by autocrine activation of TGFbeta. J Am Soc Nephrol 1999;10:1891-9.

5. Mashiko T, Abo Y, Kuno S, Yoshimura K. A novel facial rejuvenation treatment using pneumatic injection of non-cross-linked hyaluronic acid and hypertonic glucose solution. Dermatol Surg 2015;41:755-8.

6. Grinnell F. Fibroblast biology in three-dimensional collagen matrices. Trends Cell Biol 2003;13:264-9.

7. Rolin GL, Binda D, Tissot M, Viennet C, Saas P, Muret P, et al. In vitro study of the impact of mechanical tension on the dermal fibroblast phenotype in the context of skin wound healing. J Biomech 2014;47:3555-61.

8. Kessler D, Dethlefsen S, Haase I, Plomann M, Hirche F, Krieg $T$, et al. Fibroblasts in mechanically stressed collagen lattices assume a "synthetic" phenotype. J Biol Chem 2001;276:3657585.

9. Oh S, Ettema AM, Zhao C, Zobitz ME, Wold LE, An KN, et al. Dextrose-induced subsynovial connective tissue fibrosis in the rabbit carpal tunnel: a potential model to study carpal tunnel syndrome? Hand (N Y) 2008;3:34-40.

10. Yoshii Y, Zhao C, Schmelzer JD, Low PA, An KN, Amadio PC. Effects of multiple injections of hypertonic dextrose in the rabbit carpal tunnel: a potential model of carpal tunnel syndrome development. Hand (N Y) 2014;9:52-7.
11. Seok J, Oh CT, Kwon HJ, Kwon TR, Choi EJ, Choi SY, et al. Investigating skin penetration depth and shape following needlefree injection at different pressures: a cadaveric study. Lasers Surg Med 2016;48:624-8.

12. Cho SB, Kwon TR, Yoo KH, Oh CT, Choi EJ, Kim BJ. Transcutaneous pneumatic injection of glucose solution: a morphometric evaluation of in vivo micropig skin and tissue-mimicking phantom. Skin Res Technol 2017;23:88-96.

13. Kim BJ, Yoo KH, Kim MN. Successful treatment of depressed scars of the forehead secondary to herpes zoster using subdermal minimal surgery technology. Dermatol Surg 2009;35:143940.

14. Han TY, Lee JW, Lee JH, Son SJ, Kim BJ, Mun SK, et al. Subdermal minimal surgery with hyaluronic acid as an effective treatment for neck wrinkles. Dermatol Surg 2011;37:1291-6.

15. Lee JW, Kim BJ, Kim MN, Lee CK. Treatment of acne scars using subdermal minimal surgery technology. Dermatol Surg 2010;36:1281-7.

16. Agarwal CA, Mendenhall SD 3rd, Foreman KB, Owsley JQ. The course of the frontal branch of the facial nerve in relation to fascial planes: an anatomic study. Plast Reconstr Surg 2010;125:532-7.

17. O'Brien JX, Ashton MW, Rozen WM, Ross R, Mendelson BC. New perspectives on the surgical anatomy and nomenclature of the temporal region: literature review and dissection study. Plast Reconstr Surg 2013;131:510-22.

18. Breithaupt AD, Jones DH, Braz A, Narins R, Weinkle S. Anatomical basis for safe and effective volumization of the temple. Dermatol Surg 2015;41 Suppl 1:S278-83.

19. Moradi A, Shirazi A, Perez V. A guide to temporal fossa augmentation with small gel particle hyaluronic acid dermal filler. J Drugs Dermatol 2011;10:673-6. 